\title{
A Framework for Designing Closed-loop Hand Gesture Interface Incorporating Compatibility between Human and Monocular Device
}

\author{
Hyunsoo Lee, Sangho Kim
}

School of Industrial Engineering, Kumoh National Institute of Technology (KIT), Gumi, 730-701

\begin{abstract}
Objective: This paper targets a framework of a hand gesture based interface design. Background: While a modeling of contact-based interfaces has focused on users' ergonomic interface designs and real-time technologies, an implementation of a contactless interface needs error-free classifications as an essential prior condition. These trends made many research studies concentrate on the designs of feature vectors, learning models and their tests. Even though there have been remarkable advances in this field, the ignorance of ergonomics and users' cognitions result in several problems including a user's uneasy behaviors. Method: In order to incorporate compatibilities considering users' comfortable behaviors and device's classification abilities simultaneously, classification-oriented gestures are extracted using the suggested human-hand model and closed-loop classification procedures. Out of the extracted gestures, the compatibility-oriented gestures are acquired though human's ergonomic and cognitive experiments. Then, the obtained hand gestures are converted into a series of hand behaviors Handycon - which is mapped into several functions in a mobile device. Results: This Handycon model guarantees users' easy behavior and helps fast understandings as well as the high classification rate. Conclusion and Application: The suggested framework contributes to develop a hand gesture-based contactless interface model considering compatibilities between human and device. The suggested procedures can be applied effectively into other contactless interface designs.
\end{abstract}

Keywords: Contactless interface, Compatibility, Classification-oriented gesture, Compatibility-oriented gesture, Handycon

\section{Introduction}

인간과 외부기기와의 인터페이스를 설계하는 과정에서 추 구되는 최종 목표는 인간이 원하는 출력값을 기기에 (1) 정 확하고(Noiseless Transmission) (2) 빠르게(Real-time Interface) 전달함과 동시에, 그 전달과정에서 (3) 인간의 편의성을 극대화(Pursuit of Human Convenience)하는 것이다. 인간-기계 인터페이스를 접촉식(Contact-based Interface) 과 비접촉식 (Contactless Interface)으로 구분
할 때, 접촉식은 인간과 기기간에 기 정의된 프로토콜 (protocol) 의 직접적인 제어를 통하여 의사전달 오류를 최 소화할 수 있기 때문에 실시간 제어와 인간의 편의성 극대 화라는 두 가지 목적에 보다 큰 비중을 두고 있다. 그러나, 이와 달리 비접촉식 인터페이스 설계에서는 정확한 전달이 라는 첫 번째 목적이 보다 큰 우선순위를 가지고 추구될 수 밖에 없었으며, '인식(Classification)의 정확성 및 오류'의 문제를 필연적으로 내포한다는 점에서 구현상의 어려움을 지니게 되었다. 제어이론(Control theory)의 측면에서, 접 촉식 인터페이스는 Figure 1 (a)와 같은 블록 다이어그램으

Corresponding Author: Sangho Kim. School of Industrial Engineering, Kumoh National Institute of Technology (KIT), Gumi, $730-701$.

Mobile: +82-10-9502-4387, E-mail: kimsh@kumoh.ac.kr

Copyright@2012 by Ergonomics Society of Korea(pISSN:1229-1684 eISSN:2093-8462). All right reserved.

(c) This is an open-access article distributed under the terms of the Creative Commons Attribution Non-Commercial License(http://creativecommons.org/licenses/by-nc/3.0/), which permits unrestricted non-commercial use, distribution, and reproduction in any medium, provided the original work is properly cited. http://www.esk.or.kr 


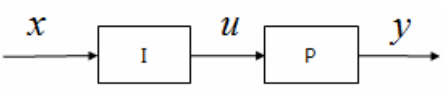

(a) Open-loop interface and control

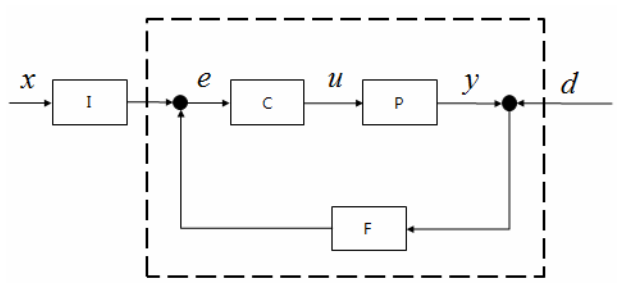

(b) Closed-loop interface and control

Figure 1. The block diagram of contact-based interface/control

$$
y=P \circ I \circ x
$$

Where, $\mathrm{P}=$ process mapping function, $\mathrm{I}=$ Interface mapping function $\mathrm{y}=$ device's output, $\mathrm{u}=$ user's interfaced value and, $\mathrm{x}=$ user's input

Figure 1(a)에 나타낸 바와 같이 사용자의 인터페이스 함 수인 I는 기 정의된 Protocol 및 구현장치에 의해 equation (2) 와 같이 $1: 1$ 함수로 맵핑되어 사용자 의도(User intent) 의 왜곡을 최소화 시키게 된다.

$$
D(u) \approx D(x)
$$

Where, $D(\cdot)$ Evaluation function for desired intent

이러한 특징은, 접촉식 인터페이스를 탑재한 기기의 feedback 설계 시 그 연결을 프로세스 맵핑 함수인 P에 집 중케 하는 효과를 나타낸다(Figure 1(b)). Figure 1(b)에서 $\mathrm{C}$ 는 control mapping function을, $\mathrm{F}$ 는 사용자의 최종 목적 값(d)를 전달하는 feedback mapping function을 각각 나 타낸다(d는 사용자가 원하는 output이며, e는 이를 얻기 위 한 입력 변수와 실제 변수 $\mathrm{x}$ 와의 차이임).

그러나, 비접촉식 인터페이스가 탑재된 기기에서는 일반적 으로 equation (2)가 보전되지 않기 때문에 $(D(u) \neq D(x))$, equation (3)과 같이 인터페이스 오류를 최소화하는 방향 으로 그 설계가 진행되고, 이를 위한 closed-loop 디자인 (Figure 2)이 필수적으로 요구된다.

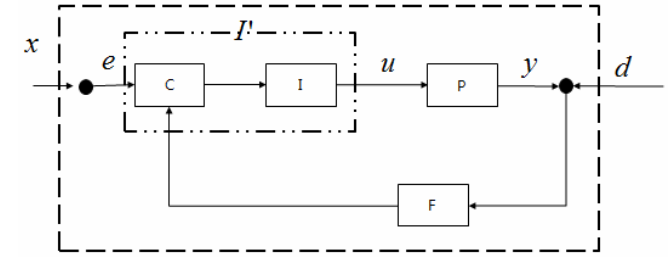

Figure 2. The block diagram of contactless interface/control

Figure 2에서 보는 바와 같이 비접촉 인터페이스의 목적 은 equation (3)을 만족하는 새로운 인터페이스(I')을 디자 인하는데 있으며, 이는 결국, Feedback 정보를 equation (3)의 기준에 맞도록, 기존 비접촉 인터페이스인 I를 대치하 는 새로운 맵핑 함수를 구현 $(C \cdot I)$ 하자는 것이다.

이로 인해 비접촉 인터페이스의 인식오류 최소화(equation (3)) 는 동시에 두 가지 목적함수를 최소화해야 하는 다목적 최적화 방법 (Multi-objective programming)의 성격을 지 니게 되는데, 첫 번째 목적함수는 기기가 사용자의 비접촉 행위를 받아들이고 이를 인식하는 과정에서 발생하는 오류 의 최소화(Type I/II 오류)이고, 두 번째 목적함수는 비접 촉 행위를 행하는 인간의 편의성 극대화로 나뉘게 된다 (equation (4)).

$$
\{\operatorname{Min}|D(u)-D(x)|, \operatorname{MIN} H(x)\}
$$

Where, $H(\cdot)=$ difficult degree of human's contactless behavior

Equation (4)의 다목적 최적화에서 각 목적함수를 수행 하는 주체는 (1) 목적함수를 충족하기 위해 각 함수들을 제 어하는 외부기기와 (2) 행위 편의성을 극대화하는 사용자 로 나눌 수 있다. 이러한 특징은 Pareto Front (Marler and Arora, 2004)를 이끌어내는 과정에서 각각의 최적화 값을 통해 전체적인 목적함수를 최적화할 수 없는 상태로 나타나 게 된다. 또한, $H(\cdot)$ 함수 자체가 정확한 수식으로 구성되 지 않는 실험값에 기초하기 때문에, 전체적인 최적화 모델을 구성할 수 없다는 특징도 지니게 된다.

따라서, 본 논문에서는 이러한 두 가지 목적함수인 외부기 기에서의 인식오류 국소화(local minimization)와 인간의 비접촉 행위 비편의성 국소화를 고려한 손의 움직임 기반 디 바이스 인터페이스의 설계를 목적으로 한다. 각 목적함수의 주체가 일치하지 않기 때문에 나타나는 충돌을 동시에 고려 하기 위해 인간과 기기간의 양립성(Compatibility)을 모형 화하고, 이를 토대로 인식이 용이하면서도 인간의 행위 편의 
성을 보장하는 움직임을 설계하고자 하였다.

이를 위해 2장에서는 본 연구와 관련된 비접촉 인터페이 스의 기술적 배경 및 기존 연구를 고찰하고, 3 장과 4장에서 는 양립성을 확보하기 위해 사용한 손의 모형 및 움직임 추 출과정을 서술하였다. 마지막으로 5장에서는, 추출된 움직임 을 기반으로 설계된, 비접촉식 모바일 기기의 제어를 위한 기능 동작의 예를 정리하였다.

\section{Background and Literature Review}

본 논문에서 연구대상으로 사용한 비접촉 인터페이스 기기는 사용자의 손 동작을 인식하여 이를 해석하고 기기를 제어하는 단일렌즈(Monocular)기반의 모바일 기기이다 (Figure 3). 현재 사용되고 있는 대부분의 모바일 기기는 한 개의 카메라 렌즈를 통하여 사용자의 손 움직임을 인지하 고 이를 기기제어의 기본 데이터로 삼고 있다.

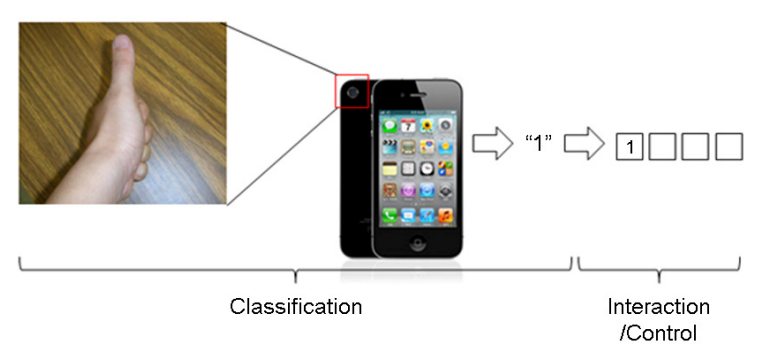

Figure 3. A hand gesture classification example in a mobile device

앞장에서도 기술하였듯이, 비접촉 인터페이스는 인식의 정 확성 구현에 가장 큰 우선순위를 두기에, 이에 대한 연구가 방대하게 진행되어 왔다. 손의 움직임 추출에 대한 연구는 주로 신호처리 및 지능형 패턴인식, 인간공학을 위시한 전자 공학, 컴퓨터공학 및 산업공학 등에서 다루어졌으며, 다양한 인식모델 및 학습을 통해 인식의 정확도를 개선하였다. 손의 움직임 인식모델에서 가장 중요한 부분은 비접촉 입력값에 대한 특징형상 (feature vector)의 추출이다. Zhang et al. (2010), Zhang and Yun(2010), Korde and Jondhale (2008)을 비롯한 수많은 연구들이 손의 움직임을 피부색 에 근거한 특징형상으로 추출하여 확률모델이나 퍼지로직 (Fuzzy Logic)으로 표현 및 인지하는 방식을 택하였다. Figure 4는 한국인이 지닌 일반적인 손의 RGB 패턴을 퍼지 로직으로 추출하여 손의 움직임만을 인식하는 일련의 과정 을 나타낸 것이다.

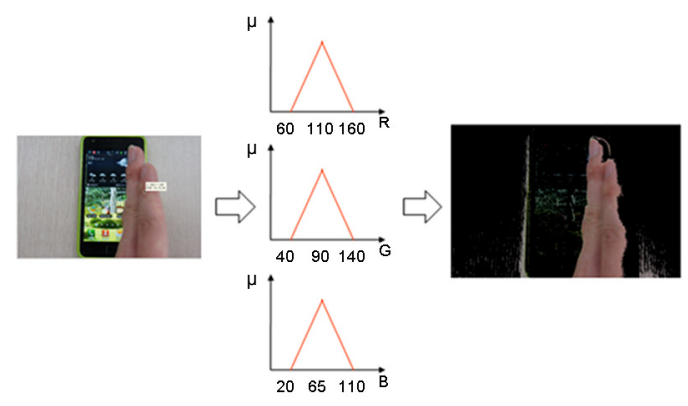

Figure 4. Fuzzy color based hand gesture recognition

손의 $\mathrm{RGB}, \mathrm{HSV}, \mathrm{YC}_{\mathrm{b}} \mathrm{C}_{\mathrm{r}}$ 값 등을 추출/변형하여 이를 이용 하는 일련의 방식들은 빠른 속도의 실시간 처리를 보장함과 동시에 비교적 정확한 인식을 보장하는 패턴인식알고리즘에 많이 이용되었다. Samson et al. (2010)은 손의 색깔을 가 지고 여성의 피부와 건강의 상관관계를 밝히는 연구에 이용 하기도 하는 등, 그 정확성 면에서 손의 색깔 관련 특징형 상은 우수하다고 할 수 있다.

그러나, 이러한 방식은 다음과 같은 면에서 단점을 지닌 다: (1) 피부색에 대한 feature vector가 인종, 장갑의 착용 및 빛의 효과 등에 의해 다르게 나타날 수 있다. (2) 손의 움직임이 아닌 타 기구에 의한 움직임은 찾아내지 못한다.

이러한 단점 등으로 인하여 특징형상은 연속된 화면 (Frame) 상에서 손의 움직임을 추적하는 Optical flow(Lin et al., 2000, Lipp, 1992) 방식이나, 초기 프레임과 연속된 프레임에서의 변화를 감지/추적하는 Background Comparison /Block Matching (Garg et al., 2009), 연속된 프레임 상 에 위치하는 물체들의 원근을 이용하는 Motion Parallax (Enzweiler et al., 2008, Hartley and Zisserman, 2004) 등 다양한 기법이 사용되거나 혼용되고 있다.

이러한 특징형상 및 기법 등은 Support Vector Machine, Back-Propagation based Neural Net, Hidden Markov Chain Model(Haykin, 1998) 등의 다양한 학습모델과 결합 되어 비접촉 인터페이스의 가장 중요한 문제 중 하나인 인식 및 기기 제어의 제 문제들을 해결하고 있다.

그러나, 인간공학적 관점에서 볼 때, 위에 기술한 방식들 은 인간의 인지적 부담이나 사용 편의성을 고려하지 못한 채 단방향적 인식문제를 다루고 있으며, 기기 제어의 측면에 서도 실시간 제어를 위해서는 많은 양의 실험 데이터 및 조 건을 요구하는 등의 문제점을 나타내었다.

이러한 문제점을 해결하기 위해 본 논문에서는 모바일 디 바이스에서의 인식률과 사용자의 인지적 사용 편의성을 동 시에 고려한 손의 움직임을 디자인하고, 이를 이용한 비접촉 인터페이스를 설계해 보았다. 이를 통해, 기기에서의 인식률 과 사용자의 편의성을 동시에 충족시킬 수 있는 비접촉 동작 
인터페이스 설계를 위한 프레임워크를 제안하였다.

다음 장에서는 비접촉 동작 인터페이스를 위한 양립성의 고려를 기술하고, 본 연구에서 사용하는 손의 움직임 모형을 설명하였다.

\section{Human Hand Model for Hand Gesture and its Compatibility}

\subsection{Human hand model for hand gesture}

본 연구에서 사용된 손의 움직임 모델은 Lu et al.(2002), Stenger et al. (2001), Lin et al. (2000), Wu et al. (2001), Rehg and Kanade(1995) 의 손의 관절모델 및 조건에 기초 하여 구성 및 변형되었다. Equation (5)는 손바닥 (palm) 과 각 손가락의 관절(joint)로 이루어진 손의 집합을 보여준다.

$$
\text { Hand }=\left\{p, \quad j_{i}^{f}\right\}_{i=0,1,2,3 \& f=0, \cdots 4}
$$

Where, $\mathrm{p}=$ palm, $\mathrm{i}=$ index for joint, and $\mathrm{f}=$ index of finger

예를 들어, $j_{1}^{0}$ 는 엄지손가락 $(\mathrm{f}=0)$ 의 2 번째 관절 $(\mathrm{i}=1)$ 을 가르킨다. Figure 5는 본 논문에서 손의 움직임을 모델링하 기 위해 사용한 손바닥 및 관절의 위치를 예시적으로 나타 낸 것이다.

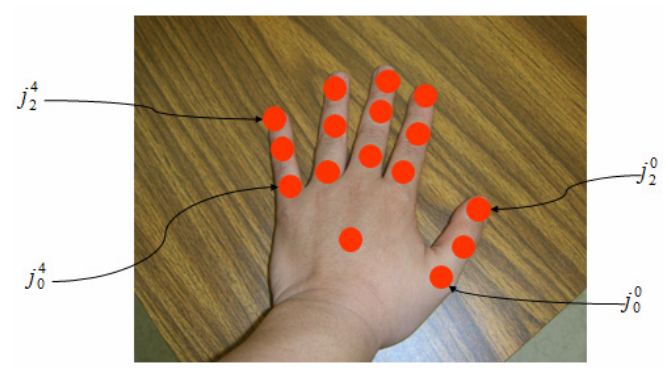

Figure 5. Human hand model for capturing hand gesture

Equation (5)에서 보여주고 있는 모델은 Lin et al. (2000) 의 연구와 같이 상호손가락 조건(Inter-finger constraint) 과 손가락내부 조건(Infra-finger constraint), 그리고 일반 움직임 조건을 가지게 되는데, 본 연구에서는 기존 연구들과 달리 비접촉 인터페이스의 확장성을 위해 상호손가락 조건 및 일반 움직임 조건만을 가지고 손의 움직임을 인식하여 모델링에 사용하였다. Table 1 은 3 단계의 관절각도로 나뉘
Table 1. Modified inter-finger constraint

\begin{tabular}{c|c|c|c}
\hline$j_{i}^{f}$ & $i=0$ & $i=1$ & $i=2$ \\
\hline$f=0$ & $\begin{array}{c}0^{\circ} / 20^{\circ} / 45^{\circ} \\
\left(-10^{\circ} \sim+45^{\circ}\right)^{*}\end{array}$ & $\begin{array}{c}0^{\circ} / 45^{\circ} / 90^{\circ} \\
\left(0^{\circ} \sim+90^{\circ}\right)\end{array}$ & $\begin{array}{c}0^{\circ} / 45^{\circ} / 90^{\circ} \\
\left(-5^{\circ} \sim+90^{\circ}\right)\end{array}$ \\
\hline$f=1 \sim 4$ & $\begin{array}{c}0^{\circ} / 45^{\circ} / 90^{\circ} \\
\left(-15^{\circ} \sim+90^{\circ}\right)\end{array}$ & $\begin{array}{c}0^{\circ} / 45^{\circ} / 90^{\circ} \\
\left(-5^{\circ} \sim+90^{\circ}\right)\end{array}$ & $\begin{array}{c}0^{\circ} / 45^{\circ} / 90^{\circ} \\
\left(-5^{\circ} \sim+90^{\circ}\right)\end{array}$ \\
\hline \multicolumn{3}{c}{$(\quad)^{*}$ Inter-finger constraint value from Lin et al. (2000) }
\end{tabular}

어진 상호손가락 조건을 보여준다.

일반적인 관절의 움직임을 3 단계로 나눈 것은 기기에서 손 동작의 특성을 인식하기에 충분한 관절 각의 수를 보여주 며, 이를 통해 틀어진 각도를 계산하는 기준(template)으로 삼을 수 있기 때문이다. 이와 더불어 사용되는 일반 움직임 조건은 $j_{2}^{f}$ 는 $j_{1}^{f}$ 을 기준으로 한 상대좌표로 계산되며, 마 찬가지로 $j_{1}^{f}$ 은 $j_{0}^{f}$ 를 기준으로 한 상대좌표로 계산된다 는 점이다.

기존의 연구들이 손의 움직임을 정확히 인식하여 복원하 는 쪽에 연구들에 주로 초점을 맞추었다면, 본 논문에서는 $2 \mathrm{D}$ 카메라 렌즈가 손의 움직임을 인식하고 기기를 제어한다 는 점에서 위의 두 가지 조건들을 가지고 비접촉 인터페이스 의 설계에 설계에 이용하는 것에 중점을 두었다.

본 논문에서는 수화(Sign Language)와 같이 양 손을 동 시에 사용하여 의사를 전달하는 방안을 고려하지 않는다. 양 손의 움직임을 동시에 인식하는 것은 겹침현상(Occlusion) 과 같은 난제와 함께, 실시간 인터액션에서 아직도 많은 연 구가 필요한 부분이다.

\subsection{Compatibility between human and device}

앞 절에서 설정한 손의 움직임 모델은 사용자와 모바일 기기간의 인식 및 제어에 관한 양립성(Compatibility)을 측정하고, 이에 기반한 손의 움직임을 설계하여 비접촉 인 터페이스의 입력으로 사용되는 제반 모형을 개발하는 과정 에서 활용되었다. 양립성이란 "기기의 제어를 위한 인식과 사용자의 인지 및 행동의 편의성을 동시에 고려하여 생성되 는 움직임 특성"으로 정의된다. 즉, 양립성을 고려한 비접촉 인터페이스 설계는 모바일 디바이스 관점에서 인식이 용이 한 손의 움직임(Classification-oriented hand gesture) 과 인간/사용자의 입장에서 취하기 쉽고 인지가 쉬운 손의 움직 임(Compatibility-oriented hand gesture)을 측정 및 비교/ 분석하여 양쪽 주체에 모두 용이한 손의 움직임만을 추출하 여 고려하는 방안이다. 다음 장에서는 각 주체에서의 양립성 체크를 위한 방안과 실험과정을 정리하였다. 


\section{Compatible Hand Gesture Design}

\subsection{Classification-oriented hand gesture}

인식이 용이한 손의 움직임은 단일 카메라 렌즈를 지닌 디 바이스 내의 인식 알고리즘 및 카메라 렌즈 자체의 특성값에 따라 다르게 설정될 수 있다. 본 논문의 목적이 기기의 인식 률을 높이기 위한 특정 알고리즘의 구현에 있지 않고, 기 구 현되어 있는 알고리즘 및 기기에서의 인식에 효율적인 손 동 작의 연구이기 때문에, 3.1절에서 수립한 손 움직임 모델을 가지고, 임의의 인식 알고리즘을 통하여 손 동작들을 추려 내었다.

Table 1 에서 보여주는 바와 같이 인간의 손은 약 $14.3 \times$ $10^{6}\left(=3^{3 \times 5}\right)$ 개의 수많은 자유도를 가진 움직임을 보여주는 데, 이 가운데 인식에 필요한 손을 디자인하기 위한 특징형 상 및 학습모델로는 Lee and Banerjee(2009)가 사용한 비 정형물체의 움직임 및 Multiple Region of Interest(ROI)에 근거한 Latent-based Support Vector Machine(LSVM) 을 사용하였다. Figure 6은 본 논문에서 사용한 LSVM을 보여준다. 입력 프레임들은 손의 회전 및 다양한 위치를 보 정하기 위해 Principle Component Analysis (PCA)기법을 이용하여 먼저 보정한 후, 순수한 관절의 움직임만을 추적할 수 있도록 디자인하였다.

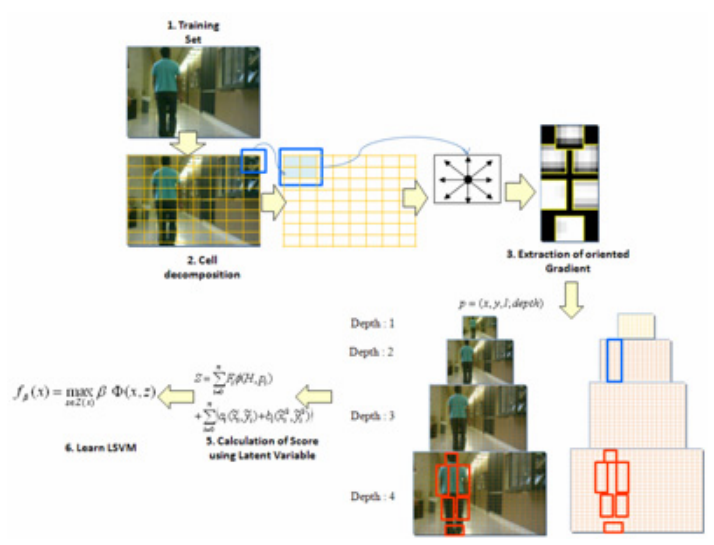

Figure 6. LSVM model(Lee and Banerjee, 2009) for classifying human hand gesture

특징형상으로는 프레임과 프레임 사이의 움직임을 Optical Flow를 사용하여 파악한 후 손바닥의 위치를 판별하고 (Primary ROI), 손의 Template 모델에 맞추어 손가락끝점 및 각 관절 (Secondary ROI)을 찾아 모델링하였다. LSVM 이 비교적 정확한 인식을 보장하지만, 본 프레임워크에서는 다른 임의의 학습모델로도 대치될 수 있도록 하였다. 이는
본 연구가 특정 디바이스에 유용한 손의 움직임이 아니라, 다수의 일반적 모바일 디바이스 프레임워크에 활용 가능한 손의 움직임을 디자인하는데 목표를 두었기 때문이다.

테스트를 위해 Virtual Hand라는 Java3D 기반의 가상 에 뮬레이터(emulator)를 구현하였고, 이 에뮬레이터를 통하여 학습 및 인식 테스트를 실시하였다. 약 $14.3 \times 10^{6}$ 개의 손 동작 중에서 500개의 테스트 샘플을 뽑아 학습시키고, 다른 임의 동작을 통하여 인식에 용이한 동작의 우선순위를 선정 하였다. Figure 7 (a)는 손 동작의 움직임 프레임을 인식하여 각 $\mathrm{ROI}$ 를 찾아내고, 이를 가상으로 복원하는 모습을 보여 주며, Figure 7 (b)는 에뮬레이터인 Virtual Hand에서의 인 식모습을 보여준다.

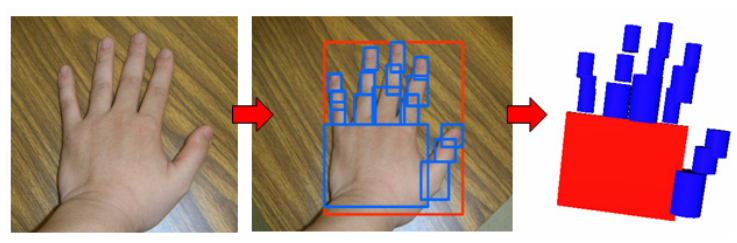

(a) Classification using monocular lens

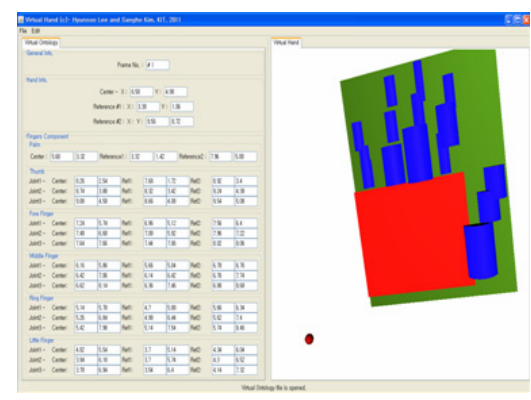

(b) Virtual Hand

Figure 7. Classification test using the emulator "Virtual Hand"

실험을 통하여 얻어진 인식에 용이한 손 움직임의 특징은 (1) 관절의 구부림 정도가 $0^{\circ}$ 에 가깝거나 $45^{\circ}$ 를 넘지 않으며 (2) 손가락의 구부러져 모아짐이 적은 움직임 (3)테스트 데 이터에 맞는 움직임(숫자 등의 표현) 그리고 (4)주먹 (fist) 과 같은 형상으로 우선순위가 설정되었다.

\subsection{Compatibility-oriented hand gesture}

앞 절을 통해 기기의 인식에 유리한 손의 움직임이 설정되 었으나, 일부 동작은 Figure 8에 예시된 것처럼 사용자가 손쉽게 따라 하기 어려운 손 동작을 포함하고 있다.

이러한 움직임에는 사람들의 인식구조에 비추어 어색한 

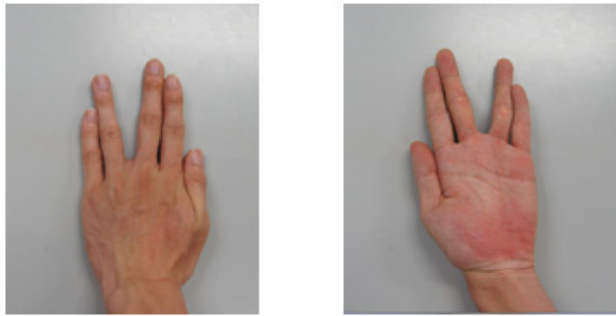

Figure 8. Several hand gesture with human's uneasy behaviors

동작들도 포함된다. 양립성 측면에서 사용자가 취하기 쉽고 인지하기에 쉬운 동작들을 추출하기 위하여 4.1절에서 추출 된 동작들을 바탕으로 손의 움직임을 실험하고 5점 척도에 맞추어 동작의 편의성 및 인지구조의 난이도를 측정하였다. 동작의 편의성은 사용자가 동작을 각각 취하여 보고, 연속 동작의 테스트를 통하여 아주 편함에서 불편함까지를 5점 척도로 측정하였고, 인식구조와 동작에 대한 연관관계의 측 정은 모바일 기기 및 일상생활에서 주로 구성되는 제어항목 들을 추출하여 동작들을 이에 맞추어 취하여 보고, 그 적정 성을 5점 척도에 맞추어 평가하였다.

이 실험을 통하여 측정된 손의 움직임은 기기에서의 인식 률과 사용자의 인지구조 및 행위 편의성이 고려된 양립성을 지닌 움직임으로 평가 받을 수 있다.

\section{Design and Evaluation of Handycon}

본 장에서는 모바일 기기의 비접촉 인터페이스에서 요구 하는 제어기능을 추출하여, 4장에서 추출된 양립성이 고려된 손의 움직임을 통해 이를 인식 및 인터페이스하는 일련의 동 작이 결합된 모형을 정리하였다. 본 연구에서는 이를 핸디콘 (Handycon)이라 명명하였다. 핸디콘의 디자인을 위하여 추 출된 손의 움직임 중 우선순위가 높은 50개를 엄선하였고, 이를 휴대용 전화기와 같은 모바일 기기의 기능과 연결하였 다. 이 중 본 절에서는 (1) 기기화면의 확대 및 축소 (2) 다 음기능 / 창으로의 전환 (3) 현 행위의 취소 (4) 현 기능/ 화면의 확인 (5) 카메라 사진 찍기 등과 같은 기능을 위해 디자인된 핸디콘의 예를 나타내었다(Figure 9).

이러한 움직임들은 Hidden Markov Chain Model(HMM) 에 의하여 모델링되어 테스트되었고, 연속화면 상에서 주어 진 기능에 맞는 동작을 인식하고 제어할 수 있게 해준다.

핸디콘은 기존의 수화와는 달리 인간의 인지구조 및 행위 편의성을 고려하는 움직임이 아니라, 기기의 Closed-loop 제어를 고려한 인식의 용이성을 갖추었다는 점에서 그 의의
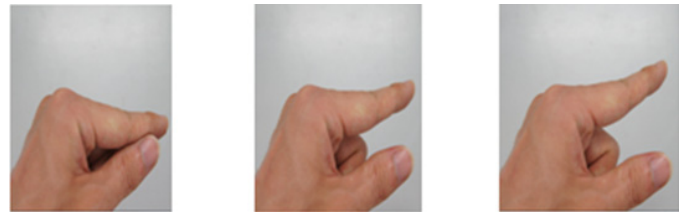

(a) Enlargement and scale-down

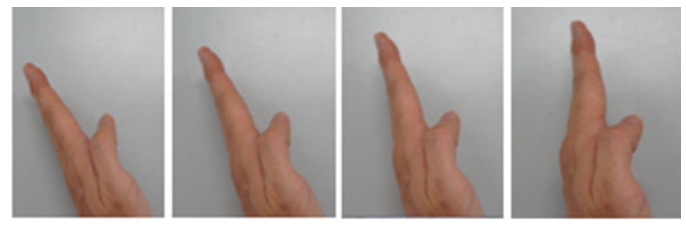

(b) Changes to next function
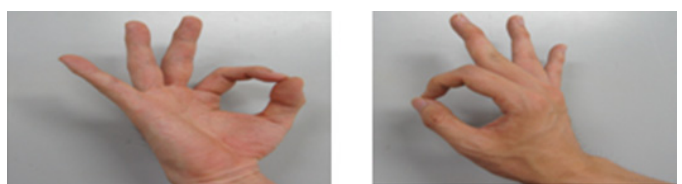

(c) Check and Selection
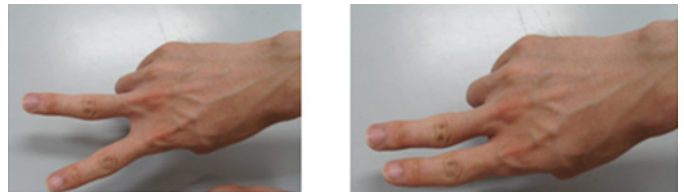

(d) Cancellation
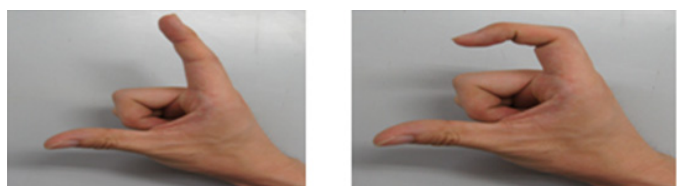

(e) Photographing

Figure 9. Examples of Handycons with compatibility

가 있으며, 이러한 동작은 손의 움직임을 인식하여 제어하는 비접촉 인터페이스의 구현을 위한 기본 입력인자로 사용될 수 있다.

Figure 9에서 보여주는 핸디콘과 기능의 맵핑은 다수의 실험대상자들의 인지 및 테스트를 통하여 추출되었으나, 실 험대상자들의 경험과 기호에 따라 타 핸디콘으로 대치될 수 있다. 이는 핸디콘을 위한 양립성 고려 시, 사용자에게 편안 한 손의 움직임 표현이 전자에 비해서 정형화 되기가 어렵다 는 점을 보여준다.

기존의 많은 연구들이 인식에 기반한 학습모델의 구성 및 발전이라는 측면에서 이루어진 반면, 핸디콘의 모델링 및 측 정은 인식과 사용자를 동시에 고려하였으며, 그 프레임워크 를 제시하였다는데 의의가 있다. 


\section{Conclusion}

본 논문에서는 단일 카메라 렌즈를 가진 모바일 기기에서 손의 움직임을 기반으로 하여 기기를 제어하는 비접촉 인터 페이스의 입력 동작을 설계하였다.

많은 연구들이 비접촉 인터페이스에서의 인식의 문제점에 초점을 맞춘 반면, 본 연구에서는 기기가 가지고 있는 임의 의 인식알고리즘 및 성능에 기초하여, 비접촉 인터페이스에 서 선결조건으로 요구되는 인식을 고려한 동작을 추출하였 고, 인간공학적 측면에서 사용성을 고려한 동작을 재추출하 였다. 이렇게 추출된 손의 동작들은 사용자와 기기를 동시에 고려한 양립성을 가지고 있으며, 이러한 손 동작을 이용하여 모바일 기기의 기능제어에 적합한 핸디콘을 설계해 보았다.

본 연구는 기기에서의 인식률이나 사용자의 편의성 중 한 쪽만을 고려함으로써 인식의 오류를 나타내거나 사용자 편 의성을 해치는 동작을 제거하고, 비접촉식 인터페이스를 통 한 기기제어 과정에 활용할 수 있는 핸디콘을 설계하는 프레 임워크를 제시하고 구현해 보았다는 점에서 의의를 가진다.

본 논문에서는 양 손을 동시에 사용하는 인터페이스에서 필수적으로 나타나는 겹침현상과 같은 이슈 등은 고려하지 않았으며, 휴대용 핸드폰뿐만 아니라, TV와 에어컨디셔너 와 같은 많은 기기에서 필요한 인터페이스 기능에서 동시에 사용될 수 있는 핸디콘의 디자인을 다루지는 않았다. 이러한 주제들은 차후 연구주제로서 다루어 질 수 있을 것이다.

\section{Acknowledgements}

This paper was supported by Research Fund, Kumoh National Institute of Technology.

\section{References}

Chaudhary, A., Reheja, J. L., Das, K. and Raheja, S., Intelligent Approaches to interact with machines using hand gesture recognition in natural way: a survey, International Journal of Computer Science and Engineering Survey, 2(1), 122-133, 2011.

Enzweiler, M., Kanter, P. and Gavrila, D. M., "Monocular pedestrian recognition using motion parallax", IEEE Intelligent Vehicles
Symposium, (pp. 792-797), Eindhoven, Netherlands, 2008.

Garg, P., Aggarwal, N. and Sofat, S., Vision based hand gesture recognition, World Academy of Science, Engineering and Technology, 49(1), 972 -977, 2009.

Hartley, R. and Zisserman, Multiple view geometry in computer vision, $2^{\text {nd }}$ Ed., Cambridge University Press, 2004.

Haykin, S., Neural networks: a comprehensive foundation, $2^{\text {nd }} \mathrm{Ed}$, Prentice Hall, 1998.

Korde, S. K. and Jondhale, K. C., "Hand gesture recognition system using standard fuzzy c-means algorithm for recognizing hand gesture with angle variations for unsupervised users", Proceedings of the 2008 First International Conference on Emerging Trends in Engineering and Technology, (pp. 681-685), Washington, DC, 2008.

Lee, H. and Banerjee, A., "Non-rigid body object tracking using fuzzy neural system based on multiple ROIs and adaptive motion frame method", IEEE International Conference on Systems, Man and Cybernetics, (pp. 1-6), San Antonio, Texas, 2009.

Lin, C. T., Chung, I. F. and Sheu, L. K. M, A neural fuzzy system for image motion estimation, Fuzzy Sets and Systems, 114(2), 281-304, 2000.

Lin, J., Wu, Y. and Huang, T. S., "Modeling human hand constraints", Proceedings of Human Motion, (pp. 1-6), Austin, Texas, 2000.

Lipp, J. I., "Frame-to-frame image motion estimation with a fuzzy logic system", Proceedings of the $35^{\text {th }}$ Midwest Symposium on Circuit and Systems, 2(pp. 987-990), 1992.

Lu, S., Huang, G., Samaras, D. and Metaxas, D., "Model-based integration of visual cues for hand tracking", Proceedings of Motion and Video Computing, (pp. 118-124), Orlando, Florida, 2002.

Marler, R. T. and Arora, J. S., Survey of multi-objective optimization methods for engineering, Structural Multidisciplinary Optimization, 26(1), 369-395, 2004

Rehg, J. M. and Kanade, T., "Model-based tracking of self-occluding articulated objects," Proceedings of ICCV, (pp. 1-6), USA, 2001.

Samson, N., Fink, B. and Matts, P., Interaction of skin color distribution and skin surface topography cues in the perception of female facial age and health, Journal of Cosmetic Dermatology, 10(1), 78-84, 2010

Stenger, B., Mendoca, P. R. S. and Cipolla, R., "Model-based 3D tracking of an articulated hand", Proceedings of CVRP, 2(pp. 310-315), Kauai, USA, 2001.

Wu, Y., Lin. J. and Huang, T. S., "Capturing natural hand articulation", Proceedings of ICCV, (pp. 426-432), USA, 2001.

Zhang, B. and Yun, R., "Robust gesture recognition based on distance distribution feature and skin-color segmentation", International Conference on Audio Language and Image Processing, (pp. 886-891), Nanjing, China, 2010.

Zhang, Q. Y., Zhang, M. Y. and Hu, J. Q., Hand gesture contour tracking based on skin color probability and state estimation model, Journal of Multimedia, 4(6), 349-355, 2009. 


\section{Author listings}

Hyunsoo Lee: hsl@kumoh.ac.kr

Highest degree: $\mathrm{PhD}$, Department of Industrial and Systems Engineering, Texas A\&M University

Position title: Assistant Professor, School of Industrial Engineering, Kumoh National Institute of Technology

Areas of interest: Artificial Intelligence, Brain Machine Interface, Intelligent Design and Control
Sangho Kim: kimsh@kumoh.ac.kr

Highest degree: $\mathrm{PhD}$, Department of Industrial Engineering, POSTECH Position title: Professor, School of Industrial Engineering, Kumoh National Institute of Technology

Areas of interest: Human Factors in Product Design, Ergonomics, Intelligent Design and Evaluation

Date Received : 2012-07-20

Date Revised :2012-07-31

Date Accepted : 2012-08-02 